\title{
O processo de cicatrização de úlceras plantares associada à terapia de contato total: uma revisão integrativa
}

\author{
The healing process of plantar ulcers associated with total contact therapy: an integrative review
}

El proceso de curación de las úlceras plantares asociada con la terapia de contacto total: una revisión integrativa

\author{
Isis Pino Maciel ${ }^{*}$, Ariane da Silva Pires², Eugenio Fuentes Pérez Júnior ${ }^{3}$, Marina Maria Bernardes \\ da Conceição ${ }^{4}$, Lidiane Passos Cunha ${ }^{5}$, Luiz Marcos Raposo ${ }^{6}$
}

Como citar esse artigo. Maciel, IP; Pires, AS; Júnior, EFP; da Conceição, MMB; Cunha, LP; Raposo, LM. O processo de cicatrização de úlceras plantares associadas à terapia de contato total: uma revisão integrativa. Revista Pró-UniverSUS. 2020 Jul./Dez.; 11 (2): $56-64$

\section{Resumo}

Introdução: Mapear a produção científica nacional e internacional relacionada a cicatrização de úlcera plantar com a utilização de terapia de contato total; e discutir as contribuições da produção científica captada para a qualidade do cuidado em Enfermagem em Podiatria Clínica. Materiais e Métodos: Revisão integrativa, que utilizou como bases de dados a Biblioteca Virtual em Saúde e PubMed. O recorte temporal deu-se entre 2012 a 2017. Resultados: Todas as produções captadas foram de periódicos internacionais, com uma incidência maior no ano de 2014, emergindo três categorias: eficácia da terapia de contato total não removível; tipos de terapia de contato total e suas comparações e contribuições para a qualidade do cuidado em enfermagem em Podiatria Clínica. Considerações finais: Propõe-se a realização de novos estudos. Fica notória a necessidade da terapia de alívio da pressão plantar para o manejo e cura da lesão, sendo o mais indicado o instant total contact cast, devido ser eficaz e ter menor chance de danos.

Palavras-chave: Úlcera plantar, Pé diabético, Cicatrização, Moldes Cirúrgicos.

\begin{abstract}
Introduction: To map the national and international scientific production related to plantar ulcer healing with the use of total contact therapy; and discuss the contributions of the scientific production captured to the quality of nursing care in Clinical Podiatrics. Materials \& Methods: Integrative review, which used as databases the Virtual Health Library and PubMed. The time frame occurred between 2012 and 2017. Results: All the productions collected were from international journals, with a higher incidence in 2014, emerging three categories: efficacy of non-removable total contact therapy; types of total contact therapy and their comparisons and contributions to the quality of nursing care in Clinical Podiatrics. Final Considerations: It is proposed to conduct further studies. The need for plantar pressure relief therapy for the management and healing of the lesion is notorious, being the most indicated the instant total contact cast, due to being effective and having a lower chance of damage.

Keywords: Foot Ulcer, Diabetic Foot, Wound Healing, Casts Surgical.
\end{abstract}

\section{Resumen}

Introducción: Mapear la producción científica nacional e internacional relacionada con la curación de úlceras plantares con el uso de terapia de contacto total; y discutir las contribuciones de la producción científica capturada a la calidad de la atención de Enfermería en Podiatria Clínica. Materiales y Métodos: Revisión integrativa, que utiliza como bases de datos la Biblioteca de Salud Virtual y PubMed. El plazo se produjo entre 2012 y 2017. Resultados: Todas las producciones recogidas fueron de revistas internacionales, con una mayor incidencia en 2014, emergiendo tres categorías: eficacia de la terapia de contacto total no extraíble; tipos de terapia de contacto total y sus comparaciones y contribuciones a la calidad de la atención de enfermería en la Clínica Podiatria. Consideraciones finales: Se propone realizar más estudios. La necesidad de terapia de alivio de presión plantar para el manejo y curación de la lesión es notoria, siendo el más indicado el contacto total instantáneo fundido, debido a ser eficaz y tener una menor probabilidad de daño.

Palabras clave: Úlcera del Pie, Pie Diabético, Cicatrización de Heridas, Moldes Quirúrgicos.

Afiliação dos autores:

${ }^{1}$ Universidade do Estado do Rio de Janeiro. Brasil. E-mail: isis.pino@gmail.com ORCID: https://orcid.org/0000-0002-9274-6875

${ }^{2}$ Doutora em Enfermagem. Docente da Faculdade de Enfermagem da Universidade do Estado do Rio de Janeiro. Brasil. E-mail: arianepiresuerj@gmail.com ORCID: https://orcid. org/0000-0003-1123-493X

${ }^{3}$ Universidade do Estado do Rio de Janeiro. Brasil. E-mail: eugenioperezjunior@gmail.com ORCID: https://orcid.org/0000-0003-4611-0443

${ }^{4}$ Universidade do Estado do Rio de Janeiro. Brasil. E-mail: marinabernardesc@hotmail.com ORCID: https://orcid.org/0000-0002-3039-3963

${ }^{5}$ Universidade do Estado do Rio de Janeiro. Brasil. E-mail: lidiane_passos_cunha@hotmail.com ORCID: https://orcid.org/0000-0001-7355-2779

${ }^{6}$ Universidade Federal de Juiz de Fora. Brasil. E-mail: luizmarcos.raposo@bol.com.br ORCID: https://orcid.org/0000-0002-5448-8369 


\section{Introdução}

As úlceras plantares, nos pacientes diabéticos, são consideradas feridas muito complexas, sentenciando os pacientes a tratamentos arrastados por longos anos, que por diversas vezes podem não levar a uma resolução do seu problema e ainda os deixar vulneráveis a infecções e amputações.

O DM e suas complicações estão se tornando rapidamente a principal causa de morbi-mortalidade do mundo. Prevê-se que, até 2040, haverá mais de 642 milhões de pessoas com diabetes no mundo ${ }^{1}$.

Pacientes com DM possuem taxa de incidência anual de úlceras plantares de $2 \%$, além do risco de $25 \%$ de desenvolvimento destas ao longo de suas vidas. $85 \%$ das amputações de membros inferiores em pacientes com DM são precedidas de úlceras, sendo os seus principais fatores de risco a neuropatia periférica (NP), as deformidades no pé e os traumatismos ${ }^{2}$.

\begin{abstract}
A NP provoca desarranjos que prejudicam os mecanismos neuro-mio-osteo-articulares, juntamente com a perda da sensibilidade protetora ocasionando um aumento do gasto energético para marcha e os pontos de hiperpressão plantar, deteriorando a qualidade deambulatória funcional e com riscos de ulceração, infecção e amputação ${ }^{3}$.
\end{abstract}

As úlceras plantares são consequência do pé diabético ${ }^{2}$ e seus fatores de riscos estão divididos em modificáveis (hiperglicemia, hipertensão, elevação dos níveis de colesterol, fumo, uso excessivo de álcool) e não modificáveis (idade avançada e diabetes de longa duração) $)^{4}$.

Essas úlceras são deveras onerosas e geram impactos importantes nas áreas econômica, social e de saúde pública, especialmente em comunidades de baixa renda que não possuam programas adequados de educação em saúde. Estima-se que o custo para o tratamento de cada úlcera plantar, no Brasil, no ano de 2009, foi de R $\$ 16.000,00^{3}$.

Mesmo com o grande avanço das práticas de saúde e conhecimento amplo da etiologia das úlceras plantares, estas podem surgir na ocorrência de falhas na prevenção associadas aos fatores de risco. Devido a isso, deve-se ter o conhecimento de várias tecnologias que possam auxiliar na cicatrização rápida e eficaz, a fim de evitar maiores riscos a esses pacientes tão vulneráveis.

A terapia de contato total (gesso de contato total) é considerada o "padrão ouro" no tratamento dessas úlceras ${ }^{5}$. As técnicas de redistribuição de carga/alívio de pressão plantar são de grande valia no tratamento das lesões, quando indicadas e aplicadas de forma correta. Existem diversas opções de terapia de descarga plantar, como: gesso de contato total, instant total contact cast, instant contact cast modificado, walker boots, pneumatic walker boots, CROW (charcot restraint orthtic walker), órteses tipo clam shell, AFO (ankle foot orthesis), sandálias de auxílio cicatricial (sandália de descarga tipo cunha), aliviadores de pressão (espumas, feltros, acolchoamentos, sandálias modificadas), auxiliadores de marcha (muletas ou andadores), cadeira de rodas e cirurgia ortopédica ${ }^{3}$.

O DM refere-se a um transtorno metabólico caracterizado por hiperglicemia crônica e distúrbios no metabolismo de carboidratos, de proteínas e de lipídios, resultantes de defeitos da secreção e/ou da ação da insulina. A hiperglicemia crônica está associada a danos e disfunções em importantes órgãos, como olhos, rins, miocárdio e vasos sanguíneos ${ }^{4}$.

Uma epidemia de DM está em curso. Hoje no Brasil, há mais de 13 milhões de pessoas vivendo com diabetes, o que representa $6,9 \%$ da população. Em alguns casos, o diagnóstico demorado favorece o aparecimento de complicações ${ }^{6}$.

A NP ocasionada pela DM é a principal causa de úlceras plantares, porém em menor frequência está também pode ser causada pelas seguintes patologias: hanseníase, sífilis, alcoolismo crônico, lesões nervosas traumáticas, doenças desmielinizantes progressivas e mielodisplasias ${ }^{7}$. Cerca de $20 \%$ dos pacientes diabéticos podem apresentar NP, que pode ser classificada em:

[...] motora (caracterizada por alteração da arquitetura do pé que desloca os sítios de pressão plantar e por alterações do colágeno, queratina e coxim adiposo); - autonômica (há disfunção simpática, resultando em redução da sudorese e alteração da microcirculação) e - sensorial (a mais comum, na qual se observa perda da sensação protetora de pressão, calor e propriocepção, de modo que traumas menores repetitivos e, até mesmo, danos maiores, não são percebidos pelos pacientes) ${ }^{8}$.

A NP provoca modificações na marcha que acaba ocasionando distribuição de pressão plantar alterada. Podem ocorrer alterações mecânicas nas articulações dos pés como deformidades em equino, luxações dos ossos sesamóides do hálux, alterações funcionais dos metatarsos, entre outras ${ }^{9}$.

Os fatores de risco para ocorrências de úlceras são a diminuição de sensibilidade e a doença vascular, também conhecida como vasculopatia diabética. Esta é dividida em duas formas: - microangiopatia, que não é considerada um fator importante na patogênese das lesões; e - macroangiopatia, que resulta em processo aterosclerótico, é mais frequente, prematuro, progressivo e mais distal, portanto, mais grave no diabético ${ }^{8,9}$.

O pé diabético é amplamente definido como "presençadeinfecção,ulceraçãoe/oudestruiçãodetecidos profundos associados a anormalidades neurológicas e a vários graus de doença vascular periférica em pessoas com DM"2, porém esta definição não aparenta ser a mais correta, visto que se deve alinhar as práticas preventivas para que não existam infecções ou ulcerações. Sendo 
assim, visando uma abrangência preventiva, conceituase como "pé diabético a presença de pelo menos uma das seguintes alterações: neurológicas, ortopédicas, vasculares e infecciosas, que podem ocorrer no pé do paciente portador de diabetes"s.

Devido às pesquisas do Dr. Paul W. Brand, um renomado cirurgião no meado do século 20 , que trabalhava com pacientes com hanseníase no sul da Índia, foi possível estabelecer a relação entre a falta de sensibilidade plantar, pressões repetitivas e a degradação da pele. Com a compreensão da patogênese das lesões dos pacientes diabéticos com NP foi possível o desenvolvimento da total contact cast (TCC ou terapia de contato total) e de outros dispositivos que realizam o alívio de pressão plantar, tendo como princípio a distribuição da carga através da superfície de contato com o solo, onde se aumenta a área de contato com o solo para haver uma diminuição da pressão vertical na região plantar, pois ela é uniformemente distribuída ${ }^{7,10}$.

As terapias de contato total evoluíram e atualmente existem opções não removíveis (tradicional) e removíveis. O modelo não removível de alívio de pressão (TCC) é moldado seguindo os contornos naturais da perna e do pé, em geral feito de gesso ou fibra de vidro, aplicado por técnico especialista. Já os removíveis (cast walker), são feitos de material mais leve, em geral plástico, associado a solado de borracha, com posibilidade de remoção pelo cliente, de forma a não atrapalhar as atividades diárias. $\mathrm{E}$ os dispositivos de alívio de pressão instantâneos não removíveis (iTCC), são formados pelo TCC removível com uma cobertura do não removível. A intenção é a de facilitar a aplicação do dispositivo, mas com o intuito de que o mesmo não seja removido pelo cliente ${ }^{11}$.

É imprescindível que exista o alívio do estresse mecânico e a redistribuição da carga de peso para a cicatrização da úlcera plantar. Estes podem ser realizados a através de calçados terapêuticos, palmilhas, órteses, gesso de contato, bota de gesso laminado, muletas, cadeiras de rodas e repouso no leito ${ }^{1,12}$. O paciente ainda pode utilizar outras modalidades que consigam manter o alívio da pressão, como um TCC, um cast walker removível, ou uma variedade de outros dispositivos, como feltros, espumas e sapatos em calha (estes últimos foram testados mas não conseguiram o alívio plantar adequado). É indubitável que haja controvérsias sobre a indicação do TCC, pois ao mesmo tempo que se afirma ser a melhor alternativa, existem riscos em sua aplicação. Posto isto, orienta-se o uso das terapias cast walker removível ou iTCC, que podem ser fabricadas em grampos de pláticos, fita adesiva ou fibra de vidro ${ }^{1}$.

Com o fito do melhor manejo do pé diabético, foi elaborado um guia de recomendações, que orienta sobre a terapia de alívio plantar e apresenta o grau de qualidade de evidência ${ }^{13}$.

Espera-se que essa pesquisa possa contribuir no âmbito do ensino - auxiliando a formação de novos profissionais e motivando a busca de conhecimento para a resolução de problemas práticos; da pesquisa - colaborando para o aumento da produção científica da temática, divulgando a expertise do enfermeiro na discussão de casos de pacientes com feridas complexas e; da assistência - apontando novas diretrizes para melhores práticas assistências, gerando mais opções no tratamento de pacientes com úlceras plantares.

Após contextualização da temática, elaborou-se a seguinte questão norteadora de pesquisa: O que há publicado nas bases de dados nacionais e internacionais acerca de cicatrização de úlceras plantares associada à terapia de contato total?

Com vistas a responder à questão norteadora, traçaram-se os seguintes objetivos: Mapear a produção científica nacional e internacional relacionada a cicatrização da úlcera plantar com a utilização de terapia de contato total; e Discutir as contribuições da produção científica captada para a qualidade do cuidado em Enfermagem em Podiatria Clínica.

\section{Metodologia}

Trata-se de um estudo bibliográfico na modalidade de revisão integrativa da literatura, que busca reunir e resumir o conhecimento científico, permitindo sintetizar as evidências disponíveis para contribuir com o desenvolvimento do conhecimento na temática. "A revisão integrativa tem o potencial de construir conhecimento em enfermagem, produzindo, um saber fundamentado e uniforme para os enfermeiros realizarem uma prática clínica de qualidade" 14 .

Para a elaboração da presente revisão integrativa as seguintes etapas foram percorridas: definição da questão norteadora (problema) e objetivos da pesquisa; estabelecimento de critérios de inclusão e exclusão das publicações (seleção da amostra); busca na literatura; análise e categorização dos estudos, apresentação e discussão dos resultados ${ }^{14}$.

Para guiar a pesquisa foi definida a seguinte questão norteadora: $\mathrm{O}$ que há publicado nas bases de dados nacionais ou internacionais acerca de cicatrização de úlceras plantares associada à terapia de contato total?

O levantamento bibliográfico foi realizado por meio de consulta à Biblioteca Virtual em Saúde (BVS) e PubMed, no mês de janeiro de 2019.

Os critérios de inclusão para composição da

Tabela 1 . Descritores cruzados nas bases de dados. Rio de Janeiro, RJ, Brasil, 2019.

BVS "úlcera plantar" OR "pé diabético" AND "cicatrização" AND "moldes" PubMed "foot ulcer" OR "diabetic foot" AND "wound healing" AND "casts"

Fonte: Pesquisa dos autores. 
produção científica para esta revisão foram: artigos científicos, publicado entre 2012 a 2018 (este recorte temporal foi selecionado com o objetivo de encontrar o que se tem de mais atual sobre a temática), estar publicado nos idiomas português, inglês ou espanhol; estar disponível na íntegra e de domínio público com versão on-line e o tema responder à questão norteadora. Os estudos que não se enquadraram nas características citadas foram excluídos desta revisão, além dos artigos em duplicidade.

Para seleção da amostra, foram utilizados os seguintes descritores "úlcera plantar", "pé diabético", "cicatrização" e "moldes" na BVS e na PubMed "foot ulcer", "diabetic foot", "wound healing" e "casts" Os descritores foram cruzados utilizando o operador booleano OR e $A N D$ (Quadro 1 - base de dados e descritores cruzados).

A coleta de dados realizou-se através de um instrumento confeccionado pela própria autora, contendo as seguintes variáveis: identificação (título, ano, periódico, país); metodologia; objetivos; principais resultados e nível de evidência.

A análise dos dados seguiu a orientação da pesquisa integrativa na qual demanda uma abordagem organizada, crítica e detalhada, a fim de analisar as características de cada estudo, seus possíveis resultados conflitantes ou diferentes e o rigor metodológico ${ }^{14}$.

Os artigos foram classificados quanto ao nível de evidência em: 1) evidências resultantes da meta-análise de múltiplos estudos clínicos controlados e randomizados; 2) evidências obtidas em estudos individuais com delineamento experimental; 3) evidências de estudos quase-experimentais; 4) evidências de estudos descritivos (não-experimentais) ou com abordagem qualitativa; 5) evidências provenientes de relatos de caso ou de experiência; e 6) evidências baseadas em opiniões de especialistas ${ }^{15}$.

\section{Resultados}

Após a busca dos artigos nas bases virtuais, foi realizada leitura e avaliação de títulos e resumos das produções. Aqueles que atenderam aos critérios previamente estabelecidos, foram selecionados para este estudo. Foi elaborado um diagrama de Prisma (Figura 1) para ilustrar o fluxo de busca e seleção do "corpus" de análise. Identificou-se um total de 37 publicações. Destas, 17 encontravam-se duplicadas. Das publicações restantes, 07 foram excluídas pois estavam fora dos critérios de inclusão. Após a leitura dos resumos, mais 04 foram excluídas, por não responderem à questão norteadora, sendo a amostra final desta revisão integrativa constituída por 09 publicações (Tabela 2).

Os artigos revisados foram publicados por oito periódicos diferentes, internacionais, indexados na
Gráfico 1 . Percentual de produção ao longo dos anos. Rio de Janeiro, RJ, Brasil, 2019.

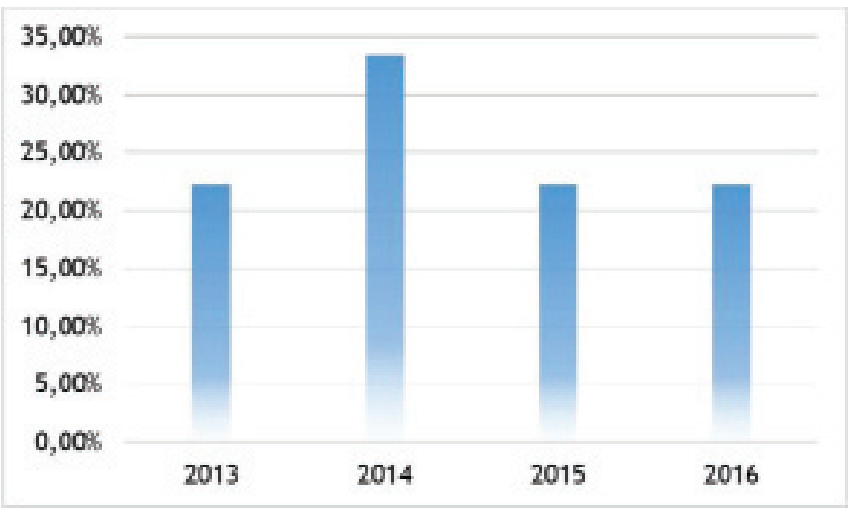

Fonte: Pesquisa dos autores.

PubMed e BVS, com exceção de um que somente foi indexado na PubMed. As publicações ficaram distribuídas entre 2013 - 2016, com uma concentração em 2014, alcançando 33,3\% do total (Gráfico 1).

Gráfico 2 . Distribuição das produções de acordo com os países. Rio de Janeiro, RJ, Brasil, 2019.

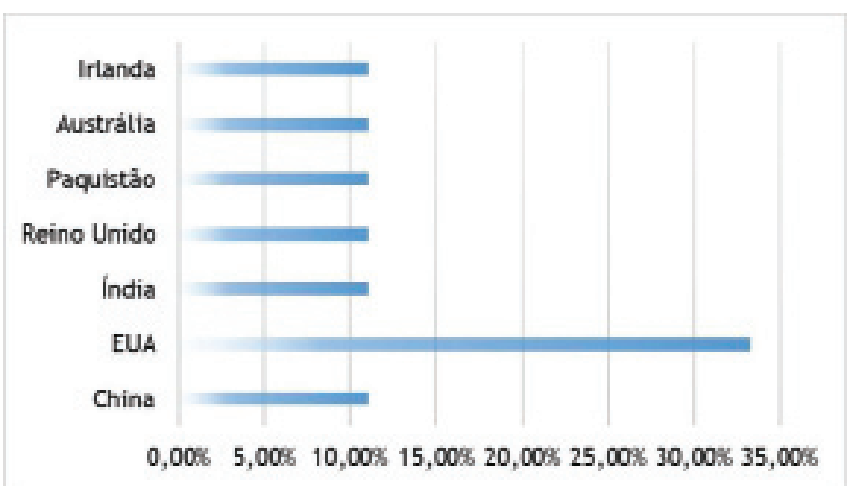

Fonte: Pesquisa dos autores.

Os Estados Unidos da América (EUA) foi o país com o maior número de publicações, apresentando três artigos (33,3\%) (Gráfico 2).

Gráfico 3 . Percentual de nível de evidência nas produções. Rio de Janeiro, RJ, Brasil, 2019.

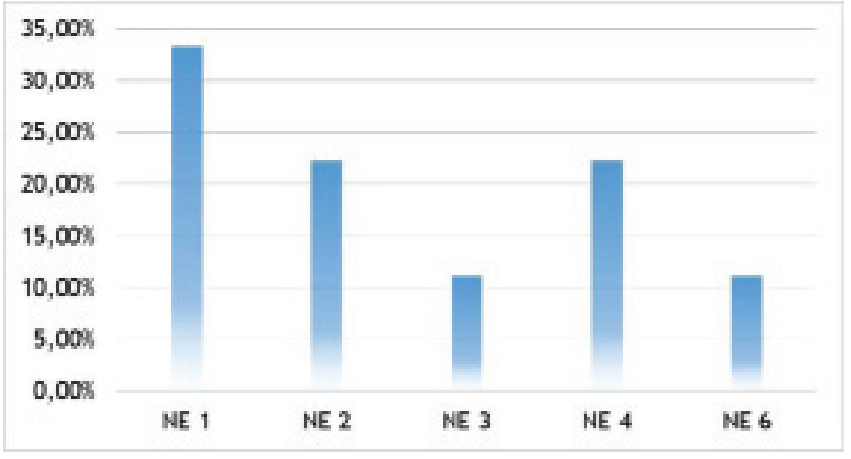

Fonte: Pesquisa dos autores. 
Tabela 2 . Estudos incluídos na revisão integrativa, segundo autor, periódico, objetivos, ano de publicação, país de origem e nível de evidência. Rio de Janeiro, RJ, Brasil, 2019.

\begin{tabular}{|c|c|c|c|c|c|}
\hline Autor & Periódico & Objetivos & Ano & País & $\begin{array}{l}\text { Nível de } \\
\text { evidência }\end{array}$ \\
\hline Wang et al16 & Med Sci Monit & $\begin{array}{l}\text { Comparar a eficiência da artrode- } \\
\text { se associada ao TCC e o TCC so- } \\
\text { zinho; para prevenção, tratamen- } \\
\text { to e recorrência de ulcerações no } \\
\text { médio-pé com neuroartropatia } \\
\text { de Charcot. }\end{array}$ & 2015 & China & 3 \\
\hline Jagadish et al17 & Am urg & $\begin{array}{l}\text { Investigar fatores que afetam a } \\
\text { taxa de cicatrização das úlceras } \\
\text { plantares do paciente diabético. }\end{array}$ & 2016 & EUA & 4 \\
\hline Rai et al18 & $\begin{array}{c}\text { Indian J Dermatol } \\
\text { Venereol Leprol }\end{array}$ & $\begin{array}{l}\text { Determinar o resultado de úlce- } \\
\text { ras plantares neuropáticas, tra- } \\
\text { tadas com descarga plantar por } \\
\text { TCC. }\end{array}$ & 2016 & Índia & 2 \\
\hline $\begin{array}{c}\text { Miller e } \\
\text { Armstrong19 }\end{array}$ & Sem Vasc Surg & $\begin{array}{l}\text { Atualização sobre as modali- } \\
\text { dades disponíveis de descarga de } \\
\text { feridas com base em pesquisas } \\
\text { lógicas sobre alívio da pressão. }\end{array}$ & 2014 & EUA & 6 \\
\hline Lavery et al11 & Int Wound $\mathrm{J}$ & $\begin{array}{l}\text { Avaliar a eficácia de três técnicas } \\
\text { de descarga plantar para curar } \\
\text { feridas de pés diabéticos: TCCs, } \\
\text { sandálias de cicatrização e uma } \\
\text { bota removível com um calca- } \\
\text { nhar de redução de cisalhamento } \\
\text { (SRB). }\end{array}$ & 2014 & EUA & 2 \\
\hline Lewis e Lipp20 & $\begin{array}{c}\text { Cochrane Database } \\
\text { Syst Rev }\end{array}$ & $\begin{array}{l}\text { Determinar os efeitos das inter- } \\
\text { venções de alívio de pressão na } \\
\text { cicatrização de úlceras plantares } \\
\text { em pessoas com diabetes. }\end{array}$ & 2013 & $\begin{array}{l}\text { Reino Uni- } \\
\text { do }\end{array}$ & 1 \\
\hline Miyan et al21 & Int Wound $\mathrm{J}$ & $\begin{array}{l}\text { Avaliar a eficácia da aplicação de } \\
\text { técnicas de alívio na úlcera plan- } \\
\text { tar em indivíduos com diabetes. }\end{array}$ & 2014 & Paquistão & 4 \\
\hline Morona et al22 & $\begin{array}{c}\text { Diabetes Metab Res } \\
\text { Rev }\end{array}$ & $\begin{array}{l}\text { Investigar a segurança e a eficácia } \\
\text { de diferentes dispositivos de des- } \\
\text { carregamento para o tratamento } \\
\text { das úlceras do pé diabético. }\end{array}$ & 2013 & Austrália & 1 \\
\hline $\begin{array}{c}\text { De Oliveira e } \\
\text { Moore23 }\end{array}$ & J Wound Care & $\begin{array}{l}\text { Comparar a literatura sobre os } \\
\text { pontos fortes e as limitações de } \\
\text { diferentes dispositivos de trata- } \\
\text { mento de úlceras no pé diabéti- } \\
\text { co. }\end{array}$ & 2015 & Irlanda & 1 \\
\hline
\end{tabular}

Fonte: Pesquisa dos autores. 
Quanto ao nível de evidência, três artigos $(33,3 \%)$ apresentaram nível 1, evidências resultantes de meta-análise e revisão sistemática; dois artigos $(22,2 \%)$ apresentaram nível de evidência 2 , evidências obtidas em estudos individuais com delineamento experimental; um estudo apresentou nível de evidência $3(11,1 \%)$, evidências de estudos quase-experimentais, dois estudos $(22,2 \%)$ apresentaram nível 4 , evidências de estudos descritivos (não-experimentais) e um artigo apresentou nível de evidência $6(11,1 \%)$, evidências baseadas em opiniões de especialistas (Gráfico 3).

Figura 1 . Diagrama de Prisma para Seleção dos Artigos. Rio de Janeiro, RJ, Brasil, 2019.

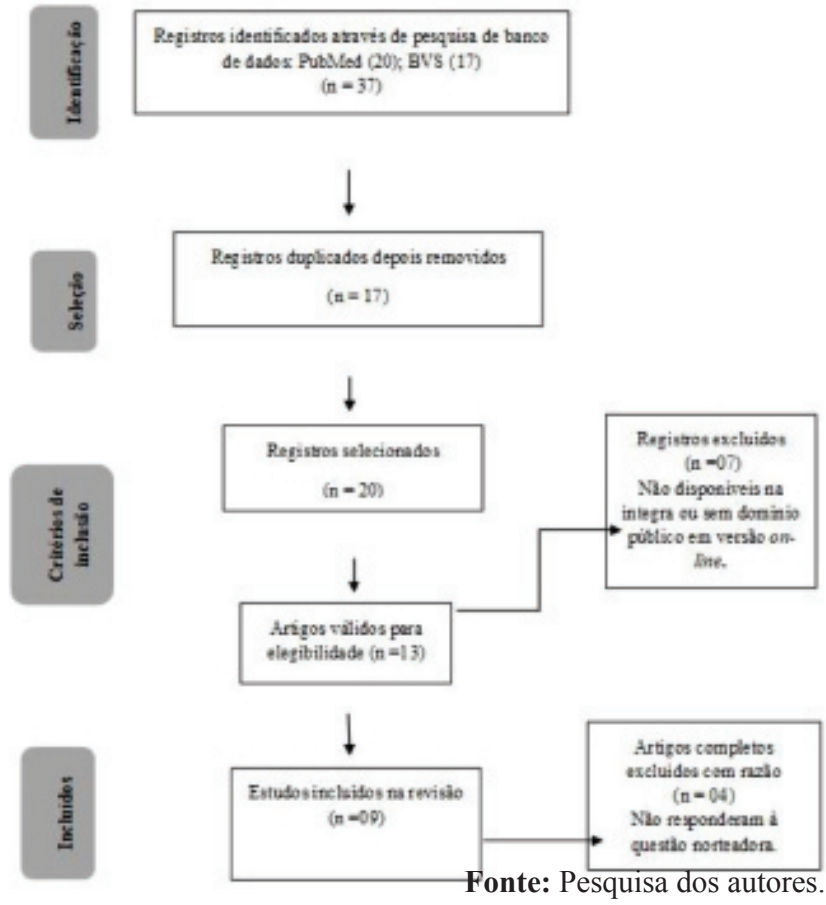

\section{Discussão}

A partir do instrumento elaborado pela autora, as informações foram condensadas em forma de tabela, permitindo, assim, a leitura e a identificação de dados relevantes obtidos a partir da literatura analisada, e, o estabelecimento da relação entre essas informações e o objeto de estudo. Após o mapeamento das obras, aplicou-se a análise temática de conteúdo em todo corpus da produção científica selecionada.

Com base nesta análise, emergiram três categorias empíricas, as quais foram construídas a partir da junção dos temas que tiveram maior incidência na literatura analisada e, intituladas como: "Eficácia da terapia de contato total não removível", "Tipos de terapia de contato total e suas comparações" e "Contribuições para a qualidade do cuidado de Enfermagem em Podiatria Clínica”.

De acordo com o material investigado, pode-se inferir que as medidas preventivas das úlceras plantares são preconizadas e fortemente incentivadas, porém muitas pessoas são acometidas por essas úlceras e necessitam das tecnologias de saúde disponíveis para o seu gerenciamento e cura.

É sabido que as técnicas de redistribuição de carga plantar pelo TCC foram divulgadas há mais de 50 anos pela sociedade científica, e vêm sendo utilizadas, testadas e aprimoradas até hoje.

\section{Eficácia da terapia de contato total não removível}

A eficácia é característica concernente de um produto/serviço ou pessoa que alcança os resultados esperados. Em suma, o material analisado mostrou uma hegemonia quanto a eficácia do TCC não removível, à medida que conseguiu seu melhor resultado, que é a cicatrização/cura da lesão plantar.

Os modelos não removíveis proporcionam a intervenção mais efetiva para aliviar as úlceras do pé diabético ${ }^{17,18,19,20}$, ajudam a controlar o edema e a deformação na fase aguda da neuroartropatia de Charcot ${ }^{19}$. Em oposição a outras modalidades de tratamento, foi associada significativamente para cura aumentada das lesões plantares, apresentando uma variação de cura de $100 \%-80 \%{ }^{17,18}$. Reforçando as recomendações das sociedades internacionais, que orientam os pacientes com úlcera plantar usar alívio com TCC ou bota de tornozelo fixo não removível, está recomendação apresenta grau de evidência $1 \mathrm{~B}$, mostrando alta confiabilidade ${ }^{13}$.

A limitação das atividades foi mencionada como promotora da cura rápida das úlceras, onde foi encontrado tempo de cicatrização de 8 a 2 semanas ${ }^{18,19}$.

Também apresenta algumas desvantagem quanto a sua aplicação, restrições de movimento e possível diminuição da qualidade de vida. Porém, quando utilizado corretamente, interrompe a cadeia de eventos na via patogênica que produz ulceração, e muda a histologia da úlcera, de um estado inflamatório crônico para uma condição mais evolutiva ${ }^{18,20}$.

Ademais, foram verificados que o tabagismo e o histórico de trombose venosa profunda, são os fatores de risco mais encontrados nos pacientes que não chegaram à cura, estando presentes em $78 \%$ e $44 \%$ respectivamente. A não cicatrização está associada ao fluxo sanguíneo diminuído e já que ambas alteram o sistema cardiovascular ${ }^{17}$. Salienta-se que a medida de maior impacto na vida da pessoa com DM é a cessação do tabagismo². 


\section{Tipos de terapia de contato total e suas comparações}

QuandosetemacomparaçãodoTCCnãoremovível com um removível e uma sandália de cicatrização, observa-se uma proporção significativamente maior de cura no grupo que utilizou o TCC não removível ${ }^{11,22}$, mesmo ele apresentado a maior taxa de desistência ao tratamento. $\mathrm{O}$ tempo de cicatrização foi menor no grupo que utilizou o TCC não removível ${ }^{11,23}$ (sendo de 5,4 a 2,9 semanas na terapia não removível, de 6,7 a 4,3 na terapia removível e de 8,9 a 3,5 no uso da sandália). Porém, quando comparado o nível de atividade, por número de passos/dia, o grupo em uso de sandália de cicatrização chegou aos maiores números $(4022$ - 4652, TCC não removível 1447 - 1310 e removível 1404 $1234)^{11}$. Mostrando com isso, que o nível de atividade tem relação com a cicatrização.

O TCC não removível necessita de técnico hábil para uma aplicação livre de iatrogenias (como piora da lesão, novas lesões, infecção de tecidos), o seu uso é não indicado nos portadores de isquemia ou infecção. Já o removível também apresenta eficácia no tratamento das lesões, é de fácil aplicação e indicado para portadores de isquemia e em pós-operatório, pois é possível o monitoramento contínuo no membro, além de possibilitar a associação de coberturas e medicamentos locais. Assim sendo, o iTCC mostra-se como um híbrido do TCC removível associado ao TCC não removível. Neste, os pacientes beneficiam-se da capacidade de alívio de pressão plantar melhor tolerada do TCC removível e a conformidade relativamente forçada do TCC não removível ${ }^{19}$.

O TCC não removível é o melhor dispositivo para alívio de pressão plantar, seguido do iTCC e da terapia removível ${ }^{22,23}$. Existe a necessidade de maiores trabalhos para confirmar se a eficácia do iTCC é igual à da terapia não removível. Ainda, relata que houve poucas evidências para apoiar a suposição que o TCC não removível está associado a uma maior incidência de efeitos colaterais e menor incidência de infecções do que dispositivos removíveis ${ }^{22}$.

Em relação ao custo, o iTCC tem menor valor (\$ 158,47 USD) do TCC não removível (\$210,67 USD) durante o curso completo do tratamento ${ }^{23}$, porém notase a necessidades de outros estudos que avaliem o custoefetividade das técnicas de alívio da pressão plantar.

Os estudos captados apresentam conformidade com a literatura buscada, esta afirma a superioridade do TCC não removível, porém indica o uso do TCC removível e do iTCC, devido sua prática evidenciar menor risco aos pacientes ${ }^{1}$.

Somente um estudo não encontrou diferença significativa em termos de eficácia de cicatrização. E expôs a necessidade de novos estudos com outras técnicas, visto que ele comparou sandália de cicatrização, modified plaster of Paris cast with plywood platform (gesso de contato total) e scotchcast boot, e em países com menores recursos ${ }^{21}$.

Quando se faz o paralelo do uso TCC não removível sozinho e seu uso após realização da artrodese de tornozelo, também não se encontram diferenças significativas em termos de cicatrização. Entretanto, foi evidenciado uma taxa superior de reincidência de úlceras plantares nos pacientes que utilizaram o TCC não removível sozinho. Ainda, pôde-se perceber que alguns pacientes recuperaram percepções sensoriais após a artrodese de tornozelo, elucidando as menores taxas de reincidência dessas úlceras nesse grupo ${ }^{16}$.

\section{Contribuições para a qualidade do cuidado de Enfermagem em Podiatria Clínica}

O cuidado de feridas está no âmago da enfermagem, porém este era realizado de forma empírica, muitas vezes não sendo valorizado.

A Podiatria Clínica nasceu no Brasil em 2007, como uma especialidade da prática do enfermeiro, prestando assistência preventiva, terapêutica e de reabilitação em busca da melhoria da qualidade de vida das pessoas com complicações nos membros inferiores ${ }^{24}$.

Este profissional apresenta como uma de suas competências clínicas o tratamento das úlceras do pé diabético. A dificuldade na execução desta tarefa é oriunda das diversas possibilidades de tratamento que devem ser meticulosamente avaliadas e discutidas com o cliente, de forma que seja encontrada uma solução que otimize a relação de custo-efetividade, apresente poucos riscos e que propicie reabilitação, autonomia e qualidade de vida.

O enfermeiro não cuida somente de úlceras e sim de pessoas. Pouco foi falado sobre o cuidado integral, não houve correlação de cessação de tabagismo, controle de pressão arterial, uso de hipoglicemiantes e controle glicêmico nos estudos captados ${ }^{2}$. Porém, o enfermeiro aborda seu cliente de maneira holística, visando o equilíbrio e qualidade de vida do portador de DM. Além disso, tamanha a complexidade do Diabetes o olhar multiprofissional se faz necessário para essa clientela e demarca o sucesso no tratamento a partir do acompanhamento do médico endocrinologista, enfermeiro, nutricionista e fisioterapeuta.

Esse cuidado diferenciado/especializado/ humanizado vem gerando uma maior credibilidade para o enfermeiro podiatra, que vem galgando seu espaço na sociedade e mostrando a necessidade dessa especialidade.

Devido ao impacto socioeconômico que esta 
enfermidade causa, o enfermeiro podiatra deve estar atualizado e inserido em pesquisas nessa temática. Este comportamento é fundamental, pois as práticas futuras tenderão a produtos com terapias inteligentes, que fornecem medições de pressão e temperatura da pele (indicadores de risco de úlcera), impressão tridimensional, auxílio na fabricação de moldes de alívio personalizados, a fim de reduzir significativamente as forças reativas do solo contra os tecidos plantares ${ }^{19}$.

Salienta-se que o alívio da pressão plantar é o elemento central em qualquer estratégia de tratamento de úlceras plantares diabéticas. A seleção de um dispositivo de descarregamento inclui eficácia, segurança, disponibilidade, custo e aceitação do paciente e conformidade ${ }^{11}$. Pautado no conhecimento advindo desse estudo o especialista pode exercer sua competência clínica com maior vigor, e prescrever uma terapia de contato total apresentando dados científicos para o seu cliente.

\section{Considerações Finais}

Conclui-se que foram atingidos os objetivos propostos. A abordagem da temática referente a cicatrização de úlceras plantares em diabéticos associada à terapia de contato total é de relevância devido ao difícil manejo dos pacientes com essas úlceras, pois envolve uma diversidade de fatores, tanto referentes ao indivíduo quanto ao gerenciamento da úlcera.

Evidenciou-se uma escassez de produção na temática apresentada, principalmente no Brasil, constatando a necessidade de realização de novos estudos comparativos, baseados em evidência e estudos custo-efetividade na realidade brasileira, para a contribuição de abordagens mais sólidas referente ao alívio da pressão plantar, que é vivenciada por muitos enfermeiros.

Considerando os resultados apreendidos, pode-se afirmar que o TCC não removível é eficaz para a cura das úlceras plantares e em menor tempo. Porém apresenta algumas desvantagens, como aplicação, restrição de movimento, maior custo e risco de iatrogenias. Sendo o TCC o dispositivo com significativa eficácia e segurança de uso, além de menor custo, fácil aplicação e possibilidade de monitoramento do membro.

Dessa forma pode-se concluir que o enfermeiro podiatra deve elencar suas condutas para uma melhoria da qualidade de vida do indivíduo focando em sua reabilitação. É necessário ter um equilíbrio em suas ações e o amplo conhecimento dos dispositivos e da pluralidade de tratamentos, com o intuito de adaptar a melhor alternativa ao cliente, avaliando riscos e benefícios.

Cabe ressaltar uma possível limitação do estudo, que foi a dificuldade para a captação dos dados, devido ao acesso restrito de algumas bases de dados, impossibilitando a obtenção gratuita de artigos relacionados ao objeto de estudo.

\section{Referências}

1. IDF. International diabetes federation. Clinical Practice Recommendation on the Diabetic Foot: A guide for health care professionals: International Diabetes Federation, 2017.

2. Brasil. Ministério da Saúde. Secretaria de Atenção à Saúde, Departamento de Atenção Básica. Manual do pé diabético: estratégias para o cuidado da pessoa com doença crônica / Ministério da Saúde, Secretaria de Atenção à Saúde, Departamento de Atenção Básica. - Brasília: Ministério da Saúde, 2016.

3. Batista, F. Uma abordagem multidisciplinar sobre o pé diabético São Paulo: Andreoli, 2010

4. Blanck M, Giannini T. Úlceras e feridas - as feridas têm alma. Uma abordagem interdisciplinar do plano de cuidados e da reconstrução estética Rio de Janeiro: Di livros, 2014.

5. SBD. Sociedade brasileira de diabetes. O que é diabetes? [homepage na Internet]. São Paulo: 2017. [acesso em 2018 Jan 28]. Disponível em: http://www.diabetes.org.br/publico/diabetes/oque-e-diabetes

6. Caiafa JS, Castro AA, Fidelis C, Santos AP, Silva ES, Sitrângulo CJ Jr. Atenção integral ao portador de pé diabético. J vasc bras [Internet]. 2011;10(4) supl.2: [aproximadamente 13 p.]. Disponível em: http://www. scielo.br/scielo.php?script $=$ sci arttext\&pid $=$ S1677-54492011000600001 [acesso em 2018 jan 20].

7. Porto BV Filho, Rodrigues GR, Cristina SK, Molina W, Amódio C, Pimenta LSM. Aplicação do gesso de contato total em úlceras neuropáticas. HSPE - Serviço de ortopedia e traumatologia - técnicas em ortopedia [Internet]. 2006;1: 17-21. Disponível em: http://tecnicasemortopedia.com. br/wp-content/uploads/2006/09/Te\%CC\%81 cnicas-em-Ortopedia-20066-1-17-21 Aplicac\% $\%$ CC $\% A 7 \mathrm{a} \% \mathrm{CC} \% 830$-do-gesso-de-contato-total-emu\%CC\%811ceras-neuropa \%CC\%81 ticas.pdf [acesso em 2017 Dez].

8. Brasileiro JL, Oliveira WTP, Monteiro LB, Chen J, Pinho EL Jr, Molkenthin S, et al. Pé diabético: aspectos clínicos. J vasc bras [Internet]. 2005; (4):1 [aproximadamente 11 p.]. Disponível em: http://jvascbras.com. br/pdf/05-04-01/05-04-01-11/05-04-01-11.pdf [acesso em 2017 Dez].

9. Cardoso VS. Estudo da marcha e de alterações biomecânicas no pé de pacientes com diabetes [dissertação]. São Paulo (SP): Programa de Mestrado em Endocrinologia, Universidade de São Paulo; 2009

10. Boulton AJM. Diabetic foot - what can we learn from leprosy? Legacy of Dr Paul W. Brand. Diabetes Metab Res Rev [Internet]. 2012 Jan; 28(Suppl 1): 3-7. Disponível em: https://onlinelibrary.wiley.com/doi/ full/10.1002/dmrr.2230[acesso em 2017 Dez]

11. Lavery LA, Baranoski S, Ayello EA. Options for off-loading the diabetic foot. Adv Skin Wound Care. 2004 May 17(4 Pt 1):181-82, 184-86. Review. Acesso em 2018 Fev 05. In: PubMed; PMID: 15360027.

12. Secretaria de Estado de Saúde do Distrito Federal. Grupo de Trabalho Internacional sobre Pé Diabético. Consenso Internacional sobre Pé Diabético/ publicado sob a direção de Hermelinda Cordeiro Pedrosa; tradução de Ana Claudia de Andrade, Hermelinda Cordeiro Pedrosa. Brasília: 2001.

13. Hingorani A, LaMuraglia GM, Henke P, Meissner MH, Loretz L, Zinszer KM, et al. The management of diabetic foot: A clinical practice guideline by the Society for Vascular Surgery in collaboration with the American Podiatric Medical Association and the Society for Vascular Medicine. J Vasc Surg [Internet]. 2016 Fev (Suppl); (63):2. Disponível em: http://www.jvascsurg.org/article/S0741-5214(15)02025-X/fulltext[acesso em 2017 Dez]

14. Mendes KDS, Silveira RCCP, Galvão CM. Revisão integrativa: método de pesquisa para a incorporação de evidências na saúde e na enfermagem. Texto Contexto Enferm. 2008 Out-Dez; 17(4): 758-64.

15. Souza MT, Silva MD, Carvalho R. Revisão integrativa: o que é e como fazer? Eintein [internet]. 2010 Jan/Mar; 8(1). Disponível em: http:// 
www.scielo.br/scielo.php?pid=S1679-45082010000100102\&script=sci arttext\&tlng=pt [acesso em 2017 Dez].

16. Wang Y, Zhou J, Yan F, Li G, Duan X, Pan H, He J. Comparison of arthrodesis with total contact casting for midfoot ulcerations associated with charcot neuroarthropathy. Med Sci Monit. 2015 Jul 24; 21:2141-8. Acesso em 2018 Fev 05. In: PubMed; PMID: 26205524.

17. Jagadish M, McNally MM, Heidel RE, Teffeteller S, Arnold JD, Freeman M, et al. Diabetic foot ulcers: the importance of patient comorbidity recognition and total contact casting in successful wound care. Am Surg. 2016 Aug; 82(8):733-6. Acesso em 2018 Fev 05. In: PubMed; PMID: 27657590 .

18. Rai S, Gupta AK, Kumar D, Aggarwal AK, Sharma VP. Total contact casting in the treatment of nonhealing plantar ulcers in anesthetic foot. Indian J Dermatol Venereol Leprol. 2016 Jul-Aug; 82(4):422-3. Acesso em 2018 Fev 05. In: PubMed; PMID: 27279305.

19. Miller J, Armstrong DG. Offloading the diabetic and ischemic foot: solutions for the vascular specialist. Semin Vasc Surg. 2014 Mar; 27(1):6874. Acesso em 2018 Fev 05. In: PubMed; PMID: 25812760.

20. Lewis J, Lipp A. Pressure-relieving interventions for treating diabetic foot ulcers. Cochrane Database Syst Rev. 2013 Jan 31;(1):CD002302. Acesso em: 2018 Fev 05. In PubMed; PMID: 23440787.

21. Miyan Z, Ahmed J, Zaidi SI, Ahmedani MY, Fawwad A, Basit A. Use of locally made off-loading techniques for diabetic plantar foot ulcer in Karachi, Pakistan. Int Wound J. 2014 Dec;11(6):691-5. Acesso em 2018 Fev 05. In: PubMed; PMID: 23369009.

22. Morona JK, Buckley ES, Jones S, Reddin EA, Merlin TL. Comparison of the clinical effectiveness of different off-loading devices for the treatment of neuropathic foot ulcers in patients with diabetes: a systematic review and meta-analysis. Diabetes Metab Res Rev. 2013 Mar; 29(3):183-93. Acesso em 2018 Fev 05. In: PubMed; PMID: 23303652.

23. de Oliveira AL, Moore Z. Treatment of the diabetic foot by offloading: a systematic review. J Wound Care. 2015 Dec; 24(12):560, 562-70. Acesso em 2018 Fev 05. In: PubMed; PMID: 26654736.

24. Portal do Enfermeiro Podiatra Brasileiro. A história da podiatria. 2020. Disponível em: < http://www.podiatria.com.br/index/historia $>$ 